\title{
Indici e Controindici: La POlemica di Pier Paolo Vergerio CONTRO La CENSURA ECClESIASTICA*
}

\section{Alessandro La MONICA}

Riassunto: In quest'articolo si esaminano alcuni dei rilievi polemici che Pier Paolo Vergerio il giovane inserì nei numerosi libelli scritti contro il papato e la Chiesa cattolica. Gli interventi vergeriani qui studiati riguardano gli Indices librorum prohibitorum, segnatamente quello veneziano del 1549, con alcuni rimandi anche a quello tridentino del 1564. L'articolo si sofferma in particolare sulla polemica che Vergerio ingaggiò contro le proibizioni di alcune opere di Dante (De monarchia), Petrarca e Boccaccio. In nota si pubblica anche una pressoché sconosciuta traduzione latina (curata da Vergerio) del sonetto petrarchesco Fontana di dolore.

Bruciatemi, scrisse di volo, bruciatemi! Questo torto non fatemelo! Non lasciatemi fuori! Che forse la verità non l'ho sempre, nei miei libri, dichiarata? E ora voi mi trattate come fossi un mentitore! Vi comando: bruciatemi!

Brecht

Nella storia italiana il numero di coloro che per motivi religiosi o politici emigrarono oltralpe, in particolare in Svizzera, è davvero considerevole. Tra i primi a cercare rifugio in quel paese, nel 1549, fu Pier Paolo Vergerio il giovane $^{1}$ (Capodistria, 1498 - Tübingen 4 ottobre 1565), arcivescovo di Capodistria poi allontanatosi dalla Chiesa cattolica il quale, dalla comunità

* Nella trascrizione del testo è stata ammodernata la grafia di apostrofi e accenti, si è distinto fra $u$ e $v$, sono state sciolte le abbreviazioni, il nesso intervocalico ti- è stato reso con -ti- o-ci-, si è eliminata l'h etimologica. Et e la sigla tironiana sono state ridotte a $e$. Regolarizzati anche l'uso delle maiuscole e l'interpunzione. Le traduzioni dal tedesco (tranne quella in epigrafe, tolta da B. Brecht, Poesie e canzoni, tr. a cura di F. Fortini e R. Leiser, Torino, Einaudi, 1959) sono dovute al sottoscritto.

${ }^{1}$ Recentemente gli studi su Vergerio hanno registrato una notevole fortuna: oltre a Schutte 1977, citiamo solo gli atti del convegno tenuto a Cividale del Friuli nell'ottobre del 1998 (Rozzo 2000). Vergerio è anche il protagonista del romanzo di Tomizza (Tomizza 1984). 
linguisticamente consorella del cantone dei Grigioni, condusse una strenua battaglia contro la censura ecclesiastica. Diverse ragioni ci spingono a soffermarci sulla sua figura: innanzitutto il rilievo internazionale del personaggio nell'ambito della contesa sulla Riforma; in secondo luogo, l'intrecciarsi della sua vicenda con quella di un'altra figura decisiva come Giovanni Della Casa; infine l'invenzione, da parte di Vergerio, del genere del controindice, sorta di Indice dei libri da leggere, come è stato autorevolmente definito da Rozzo (Pier Paolo Vergerio censore 149). Ma andiamo con ordine.

Nel 1549 l'allora nunzio pontificio di Venezia, Giovanni della Casa ${ }^{2}$ (Mugello, Toscana, 1503 - Roma 1556), famoso autore del Galateo, intraprese la compilazione del primo Index librorum probibitorum a stampa (relativo al solo territorio di Venezia) ${ }^{3}$ suscitando la reazione di Vergerio, su cui pendeva, tra l'altro, un processo per eresia intentatogli proprio da Della Casa e conclusosi con la sua condanna. Egli riparò quindi in Svizzera dove la crisi religiosa, consumata già in Italia, sfocerà nella conversione alla fede luterana e nell'inizio di una fervida attività di polemista contro gli inquisitori italiani, il Della Casa in primo luogo. Noi concentreremo in particolare la nostra attenzione su due volumi vergeriani: Il Catalogo de libri li quali nuovamente nel mese di maggio nell'anno presente M.D. XLVIIII sono stati condannati...(1549), e A gl'inquisitori che sono per l'Italia. Del catalogo di libri eretici stampato in Roma nell'Anno presente (1559). Ciò che emerge da tali scritti, a parte la vis polemica e l'efficace argomentazione, è la difesa delle "tre corone poetiche": Dante, Petrarca e Boccaccio.

Del primo e del terzo autore l'Indice del 15594 condannava rispettivamente la Monarchia e il Decameron; Petrarca, invece, citato da Vergerio come autore che avrebbe avuto tutte le carte in regola per essere colpito dalla censura, ne usciva indenne. É bene precisare, a questo punto, che appaiono fuori luogo - come ben vede Rozzo in P. P. Vergerio censore (149) - quelle interpretazioni che ascrivono tali rilievi a un presunto ruolo di delatore 5 svolto da Vergerio. In realtà egli intende solo denunciare l'incompetenza dei censori: questi, infatti, tenevano fuori dalla loro condanna opere che, sosteneva Vergerio, erano anche più pericolose di quelle incluse nel catalogo, dimostrando in tal modo di non conoscere

2 Su Della Casa vd. Santuososso 1979.

3 Per l'Indice del 1549 vd. Bujanda 1987, 383-93.

${ }^{4}$ Per l'Indice del 1559 vd. Bujanda 1990, 752-86.

${ }^{5}$ Da ultimo vd. Frajese 2006, 79-80. 
neppure l'oggetto del loro deprecabile lavoro. ${ }^{6}$

Venendo a Dante, è probabile ci fosse anche l'intervento vergeriano dietro la princeps della Monarchia, risalente al 1559. L'opera, com’è noto, da subito suscitò veementi proteste da parte ecclesiastica: già nel 1327 il domenicano Guido Vernani? scriveva un De reprobatione Monarchiae e due anni dopo il cardinale Bertrando del Poggetto (Castelnau-Montratier, 1280 circa - Villeneuve-Lès-Avignon, 1352) condannava l'opera come eretica facendola bruciare in un pubblico rogo. Fu solo dal Quattrocento che il trattato cominciò ad avere una certa fortuna, come testimoniano due volgarizzamenti: uno anonimo e l'altro, noto, a opera di Marsilio Ficino. ${ }^{8}$

Da quest'ultima versione, confrontata con un manoscritto autorevole ormai perduto, Johann Basilius Herold, ${ }^{9}$ noto umanista svizzero, ricavò la sua traduzione tedesca, pubblicata nel 1559 per "Niclaus Bischoff den jüngeren" a Basilea. Qualche settimana dopo la traduzione tedesca fu la volta dell'editio princeps pubblicata sempre a Basilea "per Joan. Oporinum". Come si ricava da alcune lettere incluse nel libro, Herold si occupò anche di questa versione. ${ }^{10}$

La domanda capitale è: chi gli fornì il manoscritto? John Tedeschi ${ }^{11} \mathrm{fa}$ il nome di Vergerio e di un altro umanista, Matthias Flacius Illyricus (Matija Vla i : Carpano, Istria, 1520 - Frankfurt, 1575) anch'egli, come Vergerio, d'origine istriana e di fede protestante.

A occuparsi più dettagliatamente della questione è stato però Francis Cheneval, nell'ambito di uno studio sulla ricezione del trattato dalle origini fino alla princeps del 1559. Da esso apprendiamo che già nel 1552-53 Flacius Illyricus cercava il manoscritto della Monarchia per una sua

${ }^{6}$ Il Catalogo, 22r: "io vi voglio far toccar con mano, che questo Legato, e questi frati sanno molto poco, e molto male sono informati da questi libri, e de questi uomini, li quali essi hanno voluto condannare, e vederete, che arrano una gran vergogna da quello, che essi hanno sperato di aver laude, e gloria”.

7 Su Vernani vd. Cheneval 1995, 78 s. (e note).

8 Per il volgarizzamento di Ficino vd. Torri 1844.

${ }^{9}$ Su Herold vd. Burkhardt 1966.

10 Lopera fu pubblicata nello stesso anno da Oporino, prima in edizione latina e poi in traduzione tedesca, curate entrambe da Herold. Si legga quanto scrive Vergerio in Della camera, et statua della Madonna chiamata di Loretto, 1554, 44r: "Dante scrisse... in prosa quel libro il cui titolo è Monarchia... e tosto lo farò io comparire in stampa".

11 Vd. Tedeschi 1987, 42, n. 13: "P. P. Vergerio e M. Flacius Illyricus contribuirono a fornire il manoscritto per la stampa". 
Kirchgeschichte e che aveva contattato fra gli altri lo stesso Vergerio perché lo aiutasse in tale ricerca. D'altro canto il futuro editore della princeps, Johannes Oporinus, già nel ' 54 era in possesso almeno di una parte del trattato, dato che ne inviò copia a un certo Windelius Rihelius, come si legge in una sua lettera del giugno dello stesso anno (Cheneval 377). Cheneval cita poi diverse opere vergeriane, dal 1554 al 1560 , da cui si ricava che l'ex arcivescovo non solo conosceva la versione di Ficino, ma era venuto in possesso, seppur con molta difficoltà, del testo originale latino. Questo almeno dichiara nel suo Postremus catalogus, un anno dopo la pubblicazione della princeps, di cui egli, tuttavia, non avrebbe saputo nulla:

Hunc ante 60 annos in Italicam linguam convertit Marsilius Ficinus, magna eruditione atque autoritate vir, cuius versionem habeo. Illud est certum, nunquam fuisse typis excusum et apud paucissimos reperiri, ego non sine difficultate potui adipisci (Vergerio, Postremus Catalogus 18).

Per cui, conclude Cheneval, non è sicuro se il manoscritto che servì per la princeps - dato anche il possesso, da parte di Oporinus, almeno di una sua parte - fosse stato procurato da Vergerio. ${ }^{12}$

Eppure il brano che Cheneval cita dal De idolo lauretano, traduzione latina del Della camera vergeriano, nell'originale italiano suona: "e tosto lo farò io comparire in stampa" ( 44 r.). Non c'è dubbio, allora, che Vergerio avesse un ruolo di primo piano nella pubblicazione della princeps, diventa, quindi, se non sicuro, certo probabile che fosse Vergerio a fornire il manoscritto; resta, semmai, incerto se il manoscritto, di cui si dichiara in possesso Oporinus nel 1554, fosse quello di Vergerio. Non essendo interessato alla polemica anticattolica, come scrive Cheneval, Oporinus forse congelò la pubblicazione del trattato, fin quando non gli proposero di pubblicare uno scritto di Alciato, il De formula Romani Imperii: l'editore allora colse l'occasione per pubblicare, insieme ad altre opere di consimile argomento, una miscellanea sulla tematica della monarchia. Quanto al

12 Vd. Cheneval 1995, 386: “... ist es nicht sicher, daß der Basler Drucker sein Manuskript von P. P. Vergerio erhalten hat, denn dieser bekundet im Jahre 1554 eine indirekte Kenntnis des Werks und bemerkt, daß nur ganz wenige dieses Opus besitzen. Im Jahre 1560 wiederholt Vergerio seine Aussage, fügt aber noch den Hinweis dazu, daß er mit großer Schwierigkeit ein Exemplar der Monarchia habe auftreiben können" [non è certo che lo stampatore di Basilea abbia ricevuto il manoscritto da Vergerio, poiché questo mostra nel 1554 una conoscenza indiretta dell'opera e rileva che solo pochissimi ne sono in possesso. Nel 1560 Vergerio ripete la sua affermazione, ma aggiunge anche che ha potuto scovare un esemplare della Monarchia con grande difficoltà.] 
fatto che la censura condannasse il trattato dantesco ben cinque anni prima della pubblicazione a stampa, non è da escludere che a ciò fosse indotta dalle citazioni di Vergerio che, come abbiamo visto, proprio nel 1554 ne annunciava un imminente edizione.

Un'opera come questa, considerato il suo autore, diventò un'arma potente in mano al fervido polemista: "Dante nel libro della monarchia la dice a lettere maiuscole, che l'imperio non depende dalla chiesa, che è tutto il contrario di quel che tenete voi" ( $A$ gl'inquisitori 30v); tuttavia, come rileveremo anche in seguito, in questo commento emerge, oltre alla polemica religiosa, sempre in primo piano, un interesse puramente letterario che rivela una profonda conoscenza dei classici. Si legga come Vergerio riprende i censori per la condanna dantesca: “... che fretta avete avuto? che furia è stata la vostra d'aver voluto in tutti i catalogi (del primo in fuori) sbandeggiar un libro il quale non fu mai stampato e trovassi molto raro?" (A gl'inquisitori 32r); e in quanto a Boccaccio Vergerio nota che gli italiani lo consideravano per la prosa "tal guida e maestro, qual è Cicerone nelle latine e Demostene nelle greche" (A gl'inquisitori 19v), né egli sembra dissentire da tale opinione.

Abile è poi il Vergerio a volgere gli argomenti dei censori contro questi ultimi, biasimandone la parzialità, non dovuta a ignoranza - come egli sapeva - ma ad opportunismo: come di Dante si condannava un trattato latino, ma s'ignoravano pour cause le rime morali, ancora più polemiche, così del Boccaccio si censuravano le cento novelle, "fatte Luterane all'improviso" (A gl'inquisitori $31 \mathrm{v}$ ), ma si glissava sulle altre opere, in volgare e in latino. Petrarca, come detto, ne esce indenne, o quasi. Infatti, non è segnalata negli Indici alcuna sua opera, se non l'edizione, a cura dello stesso Vergerio, di sedici lettere estratte dalle Sine nomine e accompagnate da tre sonetti antiavignonesi, nonostante il solo verso "Scola d'errori e tempio d'eresia"13 bastasse, sostiene Vergerio (A gl'inquisitori 33v), perché il loro autore facesse compagnia ai già condannati Dante e Boccaccio. Gli inquisitori si sono invece limitati a condannare la plaquette vergeriana salvando le opere di Petrarca, da cui quella dichiaratamente dipendeva. Ecco la spiegazione di Vergerio: "parvevi di non metter tante legne al fuoco e avendo fatto eretici Dante e Boccaccio far eretico anche il Petrarca, quantunque egli vi sia un gran stecco ne gl'occhi, specialmente essendo stato del vostro ordine. Ma non la fuggirete, che sarete costretti a dichiarirvi [sic] anche contra di lui, percioché tutte quelle sue epistole voltaremo nel volgare e in più d'una lingua" (A gl'inquisitori, 34v).

13 Il verso petrarchesco si legge in Canzoniere, CXXXVIII, 2. 
Il Canzoniere era un monumento letterario, sicché sarebbe stato assurdo vietarne la stampa; dopo la sfida vergeriana s'imponeva, tuttavia, la necessità di un'edizione espurgata dalle punte più polemiche. In realtà non si arrivò a un "Druckverbot" (Ley 250) ufficiale, ma solo implicito; come rileva giustamente Rozzo (La letteratura italiana 93), è errato sostenere che dopo la segnalazione di Vergerio le edizioni del Petrarca uscirono prive dei sonetti anticuriali; altrettanto impreciso scrivere, come fa Frajese in Nascita dell'indice - che "del Canzoniere ...furono stampate solo tre edizioni contenenti i sonetti pubblicati da Vergerio, l'ultima nel 1573" (91). Piuttosto, precisa ancora Rozzo, "le pagine con quei testi ... venivano strappate o deturpate con inchiostro in modo da renderle illeggibili" (La letteratura italiana 93): esempi di tali operazioni sono riscontrabili nelle edizioni di Venezia, Bertani, 1573, Venezia, Vidali, 1574 e Lione, Roviglio, 1574. È certo, però, che si assistette a una drastica riduzione delle stampe, riguardante non solo Petrarca, ma anche le altre opere espurgate.

Il fatto è - ribadiamo - che Vergerio, tramite la segnalazione di altre opere eminenti non incluse nell'Indice, non intendeva certo avallare la pratica censoria ma, al contrario, provocare una reazione in tutta Italia contro una condanna da lui ritenuta assurda. Di qui i riferimenti a Dante come a colui la cui autorità "specialmente nell'Italia è grandissima, come di dottissimo Filosofo e Teologo, che egli fu" (A gl'inquisitori 32r) e quelli a Boccaccio, già citati, come illustre prosatore. Petrarca, poi, non aveva, bisogno di particolari credenziali, dal momento che, specie dopo la teorizzazione bembiana, era - com'è noto - il modello unanimemente riconosciuto nel campo delle lettere. Interessante è a questo proposito l'ipotesi di Klaus Ley (246), per cui Vergerio, con paradossale rovesciamento, avrebbe rivolto quel modello proprio contro chi nel Cinquecento - dopo la morte del Bembo \pm ne era il continuatore più alto, il della Casa appunto.

Che le cose stessero così, cioè che il Nostro rilevasse numerose e importanti assenze nell'Indice non per un'inspiegabile consonanza con gli inquisitori, ma solo per provocare - dopo un'eventuale messa all'Indice delle opere da lui segnalate - una reazione indignata almeno fra i lettori, è quanto ci dice lo stesso Vergerio:

molti non arrebbono creduto, che oggidì fosse cosi grande il numero de quegli, che sono contrari alla chiesa Romana, e che fussero tanto dotti, e valenti, che sapessero contra di essa componer tanti libri, e tante opere, e quando leggerano questo catalogo [...] sarano costretti a credere, che la chiesa di Roma possa aver il torto, giacché tanti dotti uomini di tutte le 
parti del mondo sono d'accordo a scriver tanti libri contra di lei in un tempo medesimo (Il Catalogo 20v).

Lo scopo di Vergerio era dunque accrescere tale catalogo, soprattutto con autori considerati importanti in Italia. A confermare quanto andiamo dicendo è anche l'autodenuncia, altrimenti davvero inspiegabile, che Vergerio fa laddove parla dei diversi mezzi di cui gli stampatori possono servirsi per pubblicare le opere censurate:

Potrano eziandio gli stampatori, e librari alle medesime opere de Protestanti mettere in cima il nome di alcuno de Cardinali, o morti, o viventi, e così caminarano attorno secure [...] Come si è fatto di una parafrasi scritta in volgare sopra i sette salmi chiamati Penitentiali, la quale è mia, se bene è stampata sotto il nome di don Giovani da Cremona (Il Catalogo 21v).

Anche Petrarca, ancora in vita, sarebbe stato vittima della censura ad opera di un "Solipodio Dominicano" (A gl'inquisitori 34r). Di un fra Marco Piceno di Solipodio scriveva effettivamente Girolamo Squarciafico nella sua Vita Francisci Petrarchae, allegata all'edizione delle Opere latine del poeta, pubblicate a Venezia nel 1501.14 Da tale biografia Vergerio probabilmente ricavò, oltre a quest'episodio, anche l'accostamento fra i sonetti anticuriali e le Sine nomine, di cui in seguito proget tò e in parte realizzò diverse edizioni. Dalle indicazioni che Vergerio fornisce in alcuni suoi scritti, verificate con Hubert (291-2), possiamo quindi citare le seguenti edizioni a cura di Vergerio: 1) la prima in margine all'opera De idolo lauretano, in cui, come detto, si pubblicavano insieme alcuni estratti dalle Sine nomine e il sonetto Fontana di dolore in forma d'epigramma latino ${ }^{15}$; 2) una seconda edizione dell'ottobre del 1554, di sedici lettere Sine nomine, stampate, dice Vergerio, falsificando parzialmente i dati per sviare i censori,

14 Su Squarciafico vd. Frasso 1980. Per il testo della Vita Francisci Petrarchae curato da Squarciafico vd. Quarta 1909.

15 Pubblichiamo questa poco nota traduzione vergeriana, compresa in De idolo lauretano, 85: "Fons aerumnarum, domus irae, plena furoris,/ Errorum ludus, sectarum mobile templum,/ Roma quidem fueras, nunc es Babylonia fallax,/ Ex qua tot luctus, gemitusque feruntur in orbem, $/ \mathrm{O}$ fraudum mater, Carcer teterrimus irae,/ Carnificina boni, sed iniqua sedula nutrix,/ Vivorum infernus, miraclum insigne futurum/ Si nunquam contra te Christi saeviat ira./ Casta in pauperie tua sunt fundamina iacta/ Sed modo fondantes oppugnas cornibus altis./ Quid nam frontis habes meretrix? quo niteris audax/ Spes[?] In adulteriis, Spes est in divite cista./ Non igitur redeat te Constantinus adaugens/ Protinus at miserum qui sustinet aufferat orbem." 
“in Argentina da Cristiano Milio" (A gl'inquisitori 34r): in realtà si trattava di sedici lettere - come V. stesso, ricorda Hubert (294), scrive a Bullinger nel febbraio del 1455 - pubblicate a Strasburgo presso Christian Milius; 3) una terza rappresentata dalla traduzione in volgare di alcune Sine nomine accompagnate da tre sonetti antiavignonesi con il titolo Alcuni importanti luochi e, infine, 4) la raccolta Stanze del Berna con tre sonetti del Petrarca del 1554. Non solo, ma in A gl'inquisitori (34v.) Vergerio annuncia un'edizione integrale e tradotta in più lingue: "tutte quelle sue epistole [le Sine nomine di Petrarca] voltaremo nel volgare, e in più d'una lingua”.

Per quanto riguarda quest'ultima edizione, gli studiosi si dividono sulla completa o parziale falsificazione da parte di Vergerio dei testi assegnati al Berni. ${ }^{16}$ Certo è che Vergerio voleva proporre, con quest'ultimo poeta, la vicenda di una conversione esemplare — dai trascorsi giovanili licenziosi alla scoperta della vera fede, quella luterana - da opporre, intuisce bene il Ley (249) - alla biografia del Della Casa. Qui entra in gioco la questione degli scritti licenziosi e l'atteggiamento di Vergerio rispetto a essi. Ora, non c'è dubbio che egli fosse contrarissimo alle opere che "contengono cose attrative a concupiscenza carnale" (A gl'inquisitori 15v), arrivando a chiederne la censura come nel caso degli scritti dello stesso Berni, di Aretino o di Boccaccio.

Quest'ultimo rappresenta, però, un caso a sé, non integralmente condannabile per Vergerio: per due motivi, direi. Il primo riguarda l'eccellenza raggiunta dal Boccaccio nella prosa che, certificata dal Bembo, poteva far chiudere un occhio sulle oscenità presenti nel Decameron; ${ }^{17}$ il secondo, ovviamente, è rappresentato dall'importanza, per la letteratura protestante, del motivo antiecclesiastico che, com’è noto, è molto presente nelle novelle boccacciane. Vergerio ha quindi un atteggiamento ambivalente rispetto a Boccaccio: più positivo, alla fine, che negativo. ${ }^{18}$ Il commento vergeriano, infatti, si sofferma a lungo e favorevolmente sulle novelle decameroniane, ${ }^{19}$ in particolare su quelle che sono più interessanti

16 Vd. Rozzo 2005, 93-99.

17 L'autorizzazione poteva venire dalla Regola VII che precedeva l'Indice di Roma del 1564: "Antiqui [libri] vero, ab Ethnicis conscripti, propter sermonis elegantiam et proprietatem permittuntur; nulla tamen ratione pueris perlegendi erunt" (Bujanda 1990, 817).

18 Si veda ad esempio Della camera, 42v, dove Boccaccio viene definito "accorto ingegno" e viene accomunato a Petrarca e Dante, "uomini dottissimi, e di bravo ingegno... nella volgar lingua... eccellentissimi, e maestri di tutti gli altri”.

19 Come Vergerio fa anche nello scritto succitato Della camera et statua della Madonna chiamata di Loretto, 1554. 
nell'ottica protestante: la notissima novella di Ciappelletto (I, 1); quella seguente di Abraam giudeo (I, 2), aspramente critica contro la Chiesa di Roma; o, ancora, le novelle di Ferondo (III, 8), frate Puccio (III, 4), fra Cipolla (VI, 10), ecc. Non sfugge a Vergerio l'importanza che potrebbero rivestire tali novelle per la propaganda protestante e ne annuncia, infatti, un'edizione antologica con relativo commento ad opera sua e di altri collaboratori. ${ }^{20}$ Di quest' edizione, che forse non potè essere realizzata per la vastità degli interessi di Vergerio, non si ha traccia. Eppure non sarà forse fuori luogo notare che proprio in Svizzera, a Basilea in particolare, di lì a non molto sarà pubblicata una traduzione in latino proprio delle due novelle citate da Vergerio, cioè la I, 1 e la I, 2, a cura di Olimpia Fulvia Morata (Ferrara, 1526 - Heidelberg, 1555) grande figura di donna umanista che si trovava anche lei in Svizzera a quel tempo e frequentava gli ambienti protestanti, intrecciando fra l'altro una corrispondenza proprio con Vergerio. ${ }^{21}$

La letteratura oscena merita dunque, per Vergerio, una condanna senza appello, attenuata nel caso del Boccaccio per il motivo antiecclesiastico presente nel Decameron, nonché per l'alto valore della sua prosa.

Quanto al Della Casa, che ha sentito l'obbligo di "farsi un concilio in camera" (Il Catalogo 78r), Vergerio ricorda che da giovane era stato autore di non pochi versi alla maniera bernesca, fra i quali spicca scandalosamente un poemetto in cui si celebrano le lodi della sodomia. ${ }^{22}$ Vergerio allude a uno dei Capitoli pubblicati insieme a quelli del Castiglione nel 1533, intitolato Capitolo sopra il forno. In seguito Della Casa rispose, nell'opera $A d$ Germanos, ${ }^{23}$ ammettendo l'errore giovanile, ma negando che si trattasse d'amore omosessuale.

Dalle parole di Vergerio, infine, sembra sia sorto un equivoco perdu-

20 "Io conosco alcun vostro amico, il quale ha in animo di far stampare separatamente e porre in un bel libriccino tutte quelle novelle [...] e saranno forse $\mathrm{XXV}$. e speriamo che queste, essendosi aperti gl'occhi, saranno lette più volentiera, che tutte l'altre, tanto più che aremo imparato ancor noi a porvi delle postille, overo scolie nel margine e dell'annotazioni nel fine" (A gl'inquisitori 20r).

21 Si occupa della traduzione latina della novelle boccacciane Pirovano 1998.

22 Il Catalogo 78r: "In alcuni vostri versi, che sono stampati col vostro bel nome in cima, e vanno attorno insieme con $\mathrm{i}$ capitoli del Berna, voi avete tolto a celebrar le laudi... della Sodomia (questa è cosa notoria in tutta la Italia)".

23 Citiamo i vv. 22 s. di questo poemetto nella versione pubblicata da Menage: "[...] Cumque versibus/ Laudavimus Furnum, haud mares laudavimus:/ Quod ille ait per maximam calumniam:/ Sed feminas plane: ut videre Carmine/ Ex ipso adhuc potestis". 
rante ancora oggi, relativo a un altro poemetto dellacasiano che trattava lo stesso scabroso tema, ma in latino: un De laudibus pederastiae sive sodomiae. In realtà, come già nel 1725 precisava F. Menage, nel suo Anti-Baillet (449), Vergerio non vi fa mai riferimento: si è semplicemente scambiato il Capitolo del forno con quella poesia latina. Non c'è dubbio, infatti, che Vergerio si riferisca — nelle opere da noi citate — sempre al Capitolo in volgare. A tal proposito, Menage nota giustamente la ripresa implicita del v. 31 ("Benchè chi fa questo mestier divino": c.n.): "bisognava che aveste detto chiaro di condannar sua Reverendissima Signoria [Bembo], per aver sotto pretesto di cantar le Lodi del forno, celebrato le lodi del vizio pessimo e averlo chiamato opera divina e mestier divino" (A gl'inquisitori 14v: c.n.); aggiungo che anche in Il Catalogo (78r-78v) l'autore vi fa riferimento ("due volte in poche righe avete detto, che quello è un mestiere, e un'arte divina": c.n.) alludendo a un verso di poco precedente: "Soleva esser giá 'I forno un'arte santa" (11: c.n.).

Altre e più approfondite indagini merita il rapporto fra Vergerio e la censura; frattanto ci accontenteremo se, con il nostro modesto intervento, avremo suscitato la curiosità degli studiosi.

\section{UNIVERSITÀ DI ZURIGO}

\section{OPERE CITATE}

Alighieri Dante. La Monarchia di Dante Allighieri; col volgarizzamento di Marsilio Ficino; tratto dal codice inedito della Mediceo-Laurenziana di Firenze con illustrazione di diversi; a cura del dottore Alessandro Torri. Livorno: Artisti Tipografi, 1844.

Monarchey, Oder Dass das Keyserthumb, zu der wolfart diser Welt von nöten: Den Römern billich zugehört .../ Herren Dantis Aligherij des Florentiners, ein zierlichs büchlein, in drey teyl aussgeteilt...; auch neuwes verdolmetscht durch Basilium Johannem Heroldt. Basel: Bischoff, 1559.

Andreae Alciati... De formula Romani Imperii Libellus: Accesserunt non dissimilis argumenti, Dantis Florentini de Monarchia libri tres. Radulphi Carnotensis De translatione Imperii libellus. Chronica M. Jordanis, Qualiter Romanum Imperium translatum sit ad Germanos. Omnia nunc primum in lucem edita. Basileae: per Joan. Oporinum, 1559.

Burkhardt Andreas. Johannes Basilius Herold. Kaiser und Reich im protestantischen Schriftum des Basler Buchdrucks um die Mitte des 16. Jahrhunderts. Basler/Stuttgart: Helbing \& Lichtenhahn, 1967.

Bujanda, Jesùs Martinez De, ed. Index de Venice 1549, Venise et Milan 1554, Sherbrooke, Centre d'Etudes de la Renaissance.Ginevra: Droz, 1987. , ed. Index de Rome 1557, 1559, 1564. Sherbrooke, Centre d'Etudes de la Renaissance. Ginevra: Droz, 1990. 
Cheneval Francis. Die Rezeption der Monarchia Dantes bis zur Editio Princeps im Jahre 1559 : Metamorphosen eines philosophischen Werkes. München: Fink, 1995.

Frajese, Vittorio. Nascita dell'Indice. La censura ecclesiastica dal Rinascimento alla Controriforma. Brescia: Morcelliana, 2006.

Frasso G. "Cultura e scritti di Gerolamo Squarzafico." Italia medioevale e umanistica 23 (1980): 241-92.

Hubert, Friedrich. Vergerios publizistische Thätigkeit. Göttingen: Vandenhoeck \& Ruprecht, 1893.

Ley, Klaus. "Petrarca, zensiert": Aus dem Antiquariat. Zeitschrift für Antiquare und Büchersammler 4 (2004): 243-258.

Malato, Enrico. ed. Storia della letteratura italiana. Vol. 1: Dalle origini a Dante. Roma: Salerno, 1996.

Menage, Gilles. Anti-Baillet ou Critique du livre de Mr. Baillet intitulé Jugemens des Savans par Mr. Menage. Tome septieme, Premiere Partie. Amsterdam: Aux depens de la Compagnie, 1725.

Morata, Olympia Fulvia. Briefe. Leipzig: Reclam, 1991.

Pirovano, Donato. "Olimpia Morata e le prime due novelle del Decameron." Acme 51.1 (1998): 73-109.

Quarta Nino. "A proposito delle relazioni del Petrarca con Cino da Pistoia. Con nuovi documenti." Bullettino storico pistoiese 2 (1909): 42-62.

Rozzo, Ugo. ed. Pier Paolo Vergerio il Giovane, un polemista attraverso l'Europa del Cinquecento. Udine: Forum, 2000.

Rozzo, Ugo. La letteratura italiana negli Indici del Cinquecento. Udine: Forum, 2005.

Santuososso Antonio. Vita di Giovanni Della Casa. Roma: Bulzoni, 1979.

Schutte, Anne Jacobson. Pier Paolo Vergerio: The Making of an Italian Reformer. Ginevra: Droz, 1977.

Squarciafico, Girolamo. Francisci Petrarchae Florentini, philosophi, oratoris, \& poëtae clarissimi...opera quae extant omnia... Mit einer Einleitung von Johannes Herold, Texten von Hieronymus Cardanus, Giovanni Boccaccio, Erasmus, Juan Luis Vives, Francesco Florido und Paulus Jovius und einer Vita Francisci Petrarchae von Girolamo Squarciafico. Basileae: excudebat Henrichus Petri, 1554.

Tedeschi John. "I contributi culturali dei riformatori protestanti italiani nel tardo Rinascimento." Italica 64.1 (1987): 19-61.

Tomizza, Fulvio. Il male viene dal Nord: Il romanzo del vescovo Vergerio. Milano: Mondadori, 1984.

Vergerio, Pier Paolo. Il Catalogo de libri li quali nuovamente nel mese di maggio nell'anno presente M.D.XLVIIII sono stati condannati. s.l.: 1549.

. De idolo lauretano... Vergerius italice scripsit, Ludovicus eius Nepos vertit. [Tubingen: Ulrich Morhart], 1554.

- Della camera et statua della Madonna chiamata di Loretto. [Tubingen: Ulrich Morhart], 1554.

Stanze del Berna con tre sonetti del Petrarca dove si parla dell'Evangelio, ó della 
Corte Romana. [Tubingen]: 1554.

—. XVI Petrarchae epistolae. Straßburg: bei Christian Milius, 1554/5.

-. Alcuni importanti luochi tradotti fuor delle epistole latine di M. Francesco Petrarca, che fu Canonico di Padoa, archidiacono di Parma, e laureato in Campidoglio con tre sonetti, e con XVIII Stanze del Berna, che fu secretario di Papa Clem. VII. Ove vedessi che opinione hebber ambidue della Rom. Chiesa. s.l.: 1557.

A gl'inquisitori che sono per l'Italia. Del catalogo di libri eretici stampato in Roma nell'Anno presente. [Pforzheim: Corvinus (Georg Rab),]: 1559.

- Postremus catalogus haereticorum Romae conflatus 1559 continens alios quatuor catalogos qui post decennium in Italia nec non eos omnes qui in Gallia et Flandria post renatum Evangelium fuerunt aediti cum annotationibus Vergerij, Pforzheim: Corvinus (Georg Rab), 1560. 